\title{
Evolving the discussion
}

\author{
As we enter 2009 and celebrate the anniversaries associated with the birth of Darwin and publication of \\ The Origin of Species, it's worth asking why there isn't greater public awareness of the increasing molecular \\ evidence relevant to evolution and what can be done to address this.
}

$I^{\mathrm{n}}$ $\mathrm{n}$ high school chemistry class, we are all made aware of the unquestionable insight of the great Russian chemist Mendeleev. The genius of Mendeleev, who organized the elements on the basis of unifying properties and atomic weight, thus generating a 'periodic table of elements', is discussed as fact. The table predicted the existence of as-yet-undiscovered elements and, unbeknownst to its formulator, would prove to reflect the very organization of an atom.

One hundred and fifty years ago this year, Darwin published The Origin of Species, his grand argument for the principles of evolution based on descent with modification and natural selection of favorable variants. Analogous to Mendeleev's work, the theory was formulated in the absence of fundamental principles that were yet to come to light in his field. Genetic analysis would ultimately provide proof of the inheritance of variation and thus the basis for passing on variation to offspring. Further support for the concept of natural selection-evidence for variation in the form of mutations and polymorphisms, the notion of common ancestry as corroborated by DNA sequence similarities between organisms, and an explosion of evidence from areas such as structural, molecular and developmental biology—were all in the distant future. And while discussion of the details continues, these subsequent analyses fit well within the general framework formed by the idea of natural selection of variation in the population.

On page 63 of this issue, Rothenburg and colleagues have examined the principle of selective pressure in the context of the ongoing battle between host and virus. Protein kinase R (PKR) is one of a family of kinases, all of which target the same substrate, the translation-initiation factor eIF $2 \alpha$. The phosphorylation of eIF $2 \alpha$ by these kinases essentially shuts down translation under conditions of stress or, in the specific case of PKR, infection by viruses that produce double-stranded RNA as part of their life cycle. By shutting down translation upon detection of such double-stranded RNA, PKR effectively thwarts the virus. Rothenberg and colleagues have found, through comparison of PKR sequences across vertebrates, that the PKR sequence is evolving much faster than related kinase families, finding signatures indicating that it is under selective pressure. The residues under positive selection, when mapped onto the PKR structure, cluster in regions targeted by a PKR-specific pseudosubstrate inhibitor produced by poxviruses. Thus, it seems that PKR is rapidly evolving to evade such virally produced inhibitors, while retaining its enzymatic function. By replacing a single amino acid under selection in mouse PKR with the human variant (and vice versa), the authors altered susceptibility to the poxvirus-encoded PKR inhibitor K3L in a cell assay that monitored eIF $2 \alpha$ phosphorylation, providing support for the idea that these residues are evolving in different organisms in response to the inhibitors being produced by poxviruses.
As such beautiful examples of evolution in action continue to accumulate, it is worth asking why a recent online survey, conducted by the Harris polling organization in November 2008, found that only $47 \%$ of adult Americans polled believed in evolution (this compares to $40 \%$ believing in creationism, $75 \%$ in miracles and $44 \%$ in ghosts). Why, despite evolution being a relatively well-covered topic in terms of popular science writing, do the molecular data supporting natural selection remain in the realm of scientists and largely unconveyed to the public? In fact, why is there an increasingly huge gap between discovery and knowledge gained by scientists and the passing on of this information to nonscientists? It is this gap that can be exploited by those who would argue that the 'jury is out' on a given scientific topic.

Of course, the obvious discussion arising from these questions is about science education and the issue of ensuring that students have a good grounding in how science is conducted, scientific knowledge and reasoning, as well as the relationship of such an education to competitiveness. These topics are highly relevant and have previously been discussed in these pages and elsewhere. But learning about the ever-advancing scientific endeavor and research should not stop once a given student graduates from high school and college. This brings us to the important role of the media in conveying the ever-expanding wealth of information to the public at large. On 3 December 2008, the cable news network CNN caused a furor in the blogosphere when it announced that it would cut its entire unit covering science, space, technology and the environment. Their spokesperson outlined the editorial rationale underlying this decision, pointing out that the "Planet in Peril" series would cover environmental reporting, and the news agency retains a separate medical reporting staff.

Although there are a number of notable bastions of scientific reporting out there, and $\mathrm{CNN}$ was not really reporting basic science on the regular basis it deserves anyway, this move remains a measure of how increasingly marginalized science coverage seems to be becoming. This is a far cry from our idea of the days of Darwin, when scientific debate and advances were topics consumed by general newspaper readers as well as gentlemen (and lady) scientists. As we enter 2009, the year of the Darwin anniversaries, there is a golden opportunity for scientists to discuss and explain their ideas and research to the public, as well as for the media to more fully cover the evidence, including the molecular data, that continues Darwin's work. Perhaps then Darwin's concept of evolution, and the ensuing research, can be discussed in a scientifically informed fashion without reservations, and it would be clearer to the public at large why the great evolutionary biologist Dobzhansky went so far as to say, "Nothing in biology makes sense except in the light of evolution." 